\title{
Genomic heterozygosity and hybrid breakdown in cotton (Gossypium): different traits, different effects
}

\author{
Baosheng Dai, Huanle Guo, Cong Huang, Xianlong Zhang and Zhongxu Lin*
}

\begin{abstract}
Background: Hybrid breakdown has been well documented in various species. Relationships between genomic heterozygosity and traits-fitness have been extensively explored especially in the natural populations. But correlations between genomic heterozygosity and vegetative and reproductive traits in cotton interspecific populations have not been studied. In the current study, two reciprocal $F_{2}$ populations were developed using Gossypium hirsutum cv. Emian 22 and G. barbadense acc. 3-79 as parents to study hybrid breakdown in cotton. A total of 125 simple sequence repeat (SSR) markers were used to genotype the two $F_{2}$ interspecific populations.

Results: To guarantee mutual independence among the genotyped markers, the 125 SSR markers were checked by the linkage disequilibrium analysis. To our knowledge, this is a novel approach to evaluate the individual genomic heterozygosity. After marker checking, 83 common loci were used to assess the extent of genomic heterozygosity. Hybrid breakdown was found extensively in the two interspecific $F_{2}$ populations particularly on the reproductive traits because of the infertility and the bare seeds. And then, the relationships between the genomic heterozygosity and the vegetative reproductive traits were investigated. The only relationships between hybrid breakdown and heterozygosity were observed in the (Emian22 $\times 3-79) \mathrm{F}_{2}$ population for seed index (SI) and boll number per plant (BN). The maternal cytoplasmic environment may have a significant effect on genomic heterozygosity and on correlations between heterozygosity and reproductive traits.

Conclusions: A novel approach was used to evaluate genomic heterozygosity in cotton; and hybrid breakdown was observed in reproductive traits in cotton. These findings may offer new insight into hybrid breakdown in allotetraploid cotton interspecific hybrids, and may be useful for the development of interspecific hybrids for cotton genetic improvement.
\end{abstract}

Keywords: Genomic heterozygosity, Hybrid breakdown, Gossypium, Linkage disequilibrium

\section{Background}

Species divergence is currently an area of intense study [1, 2]. One related topic that has been received an increasing amount of attention is the examination of phenotypes expressed by hybrids between species [3]. Hybrid breakdown is the loss of fitness in hybrids between species, showing as inferior viability and fertility in $F_{2}$ and later generations [3]. Hybrid breakdown can be viewed as an indicator of an early stage in the evolution of a new species, so this phenomenon may provide clues into the

\footnotetext{
* Correspondence: linzhongxu@mail.hzau.edu.cn

National Key Laboratory of Crop Genetic Improvement, Huazhong

Agricultural University, Wuhan 430070, Hubei, China
}

genetics of speciation [4]. Hybrid breakdown has been well documented in both plant and animal hybrids, such as in the inter-subspecific $O$. sativa ssp. japonica $\times$ ssp. indica hybrid [5, 6], and the hybrid parasitoid wasp genus Nasonia [7]. Heterozygosity-fitness correlations have been used to study the relationships between genomic heterozygosity and fitness-related traits at the individual level in a variety of organisms [8-12].

Several alternative genetic explanations for the prevalence of hybrid breakdown have been reported in recent studies including Bateson-Dobzhansky-Muller (BDM) incompatibilities [13], the incompatibilities between the nuclear genome and the organellar genomes of mitochondria and chloroplasts [4], and disruption of co-adapted 
gene complexes [14]. The BDM model of incompatibility involves a deleterious epistatic interaction between alleles at two different loci affecting the descendant of the interspecific hybrid as much as an inter-subspecific hybrid. Several pairs of epistatic alleles are responsible for hybrid breakdown between japonica and indica cultivars of rice, which have been mapped to specific genomic regions [3, 5, 15]. The hybrid breakdown of Arabidopsis hybrids has been ascribed to BDM incompatibility involving reciprocal silencing of duplicated genes [16].

The molecular mechanisms of hybrid breakdown underlying nucleo-cytoplasmic genomic interactions have been well demonstrated [4]. Given the co-evolution of the organellar genomes and the nuclear genome, the disruption of inter-genomic coadaptation can result in organelle dysfunction and consequent hybrid breakdown. The fitness loss in marine copepod Tigriopus californicus hybrids is completely attributable to nuclear-mitochondrial genomic interactions which led to reduced ATP synthesis [17]; the nuclear-cytoplasmic data revealed an increased tendency towards maladaptation in inter-population crosses [14].

The theory of co-adapted gene complexes suggests that gene combinations are co-adapted if high fitness depends on specific interactions between them; such gene combinations are referred to as co-adapted gene complexes [18]. A consequent loss of heterosis in Drosophila hybrid populations was ascribed to breakdown of co-adapted gene complexes [19].

Although several theories have been used to explain the genetic causes underlying hybrid breakdown, heterozygosity-fitness correlations have rarely been studied in crop plants. Nevertheless, various evolutionary biology studies have examined relationships between individual genomic heterozygosity and fitness using heterozygosity-fitness correlations [20-24]. Individual genomic heterozygosity is usually estimated using neutral genetic markers, such as simple sequence repeats (SSRs) or single-nucleotide polymorphisms (SNPs). Two hypotheses have been used to explain heterozygosity-fitness correlations in interspecific hybrids: outbreeding depression [25] and local effects caused by functional genes neighbouring neutral markers, which led to the observed correlations [26].

Relationships between genomic heterozygosity and traitfitness have been extensively explored because they have strong implications for ecology and evolution. Heterozygosity-fitness correlations have been used to study relationships between genomic heterozygosity and fitness-related traits at the individual level in natural hybrid populations of a variety of organisms [8-12]. Assessment of individual genomic heterozygosity has a very important role in determining heterozygosity-fitness correlations. Several studies have utilized a molecular hybrid index (MHI) to measure genomic heterozygosity $[24,27,28]$. To increase the accuracy when calculating genomic heterozygosity, the effects of marker number and marker type have been studied [24, 27, 29]. Miller et al. [27] argued that SNPs performed similarly to microsatellites in terms of precision and accuracy in genomic heterozygosity calculations.

The genus Gossypium includes four important cultivated species, G. hirsutum, G. barbadense, G. arboreum and G. herbaceum [30]. Each species has unique advantageous traits that are imperative for cotton breeding. To combine the advantages of each, interspecific crossings between $G$. hirsutum and G. barbadense have been performed extensively [31]. The cross between the allotetraploid cottons is straightforward and can produce vigorous fertile $F_{1}$ hybrids; but serious segregation occurs in later generations, which contain many weak, and even infertile plants [32, 33], known as hybrid breakdown. Stephens found that selective elimination of a donor parent genotype, detected in interspecific backcrosses involving G. hirsutum and G. barbadense, was the likely cause of the breakdown [33].

Correlations between genomic heterozygosity and vegetative and reproductive traits in cotton interspecific populations have not been studied. In the current study, we developed reciprocal $F_{2}$ populations derived from the cross between G. hirsutum cv. Emian 22 (denoted as E22) and G. barbadense acc. 3-79 (denoted as 3-79), including a direct cross $(E 22 \times 3-79) F_{2}$ population (denoted as $\left.(E 3) F_{2}\right)$ and a reciprocal cross $(3-79 \times \mathrm{E} 22) \mathrm{F}_{2}$ population (denoted as (3E) $F_{2}$ ). This mating design allowed us to investigate maternal effects. Here, we first present an improved approach to evaluate individual genomic heterozygosity. Second, we investigate the distribution of individual genomic heterozygosity in interspecific $F_{2}$ populations of allotetraploid cottons. Third, we investigate hybrid breakdown of vegetative and reproductive traits in reciprocal $F_{2}$ populations. We also investigate the relationships between genomic heterozygosity and vegetative and reproductive traits.

\section{Methods \\ Plant materials}

The plant materials, G. hirsutum cv. Emian 22 (E22) and G. barbadense acc. 3-79 were collected for scientific research from Huanggang Academy of Agricultural Sciences (HGAAS, Huanggang, China) and the Institute of Cotton Research, Chinese Academy of Agricultural Sciences (CAAS, Anyang, China), respectively. E22 is an elite upland cotton cultivar developed by HGAAS and available in China; it was approved by the National Crops Variety Approval Committee (China) in 2000, and the authorized number was "Guoshenmian 20000006". G. barbadense acc. 3-79 is the genetic and cytogenetic standard line of G. barbadense and worldwidely available; it was identified by Kohel RJ [34]. The two cotton materials are not the specimens deposited in a herbarium and deposited in the Group of Cotton Genetic Improvement (GCGI) of 
National Key Laboratory of Crop Genetic Improvement (China).

E22 and 3-79 were used as parents to develop $F_{2}$ segregating populations. To investigate maternal effect, we constructed an $\mathrm{F}_{2}$ population with 142 individuals from an E22 (female parent) $\times 3-79$ (male parent) direct cross, as well as an $F_{2}$ population with 142 individuals from a 3-79 (female parent) $\times$ E22 (male parent) reciprocal cross. The benefit of this experimental design is that distributions of individual genomic heterozygosity can be observed in two different maternal cytoplasmic backgrounds under the same genomic background. This strategy allows us to distinguish effects from different maternal cytoplasmic backgrounds on the relationships between genomic heterozygosity and vegetative and reproductive traits.

\section{Phenotyping vegetative and reproductive traits}

The reciprocal $F_{2}$ populations together with their parents, E22 and 3-79, were grown and evaluated in the cotton breeding station at Huazhong Agricultural University, Wuhan, China in 2013. Space between the plants was maintained at $35 \mathrm{~cm}$ within the rows and at $100 \mathrm{~cm}$ between rows, and plant density was maintained at approximate 27500 plants ha $^{-1}$.

To investigate hybrid breakdown in these interspecific crossing populations, two plant vegetative traits: plant height $(\mathrm{PH})$ and branch number $(\mathrm{BrN}))$ and three reproductive traits: boll number (BN), seed set weight(SW) and seed index (SI) were chosen according to the life history of cultivated cotton. Since cultivated cotton is annual crops, the plant height and branch number in the boll-forming stages could generally reflect the vegetative viability; the boll number, seed set weight and seed index could generally reflect the reproductive fertility. Two plant vegetative traits, $\mathrm{PH}$ and $\mathrm{BrN}$, were investigated on August 15 before pinching. Total BN was explored on September 15 before the approach of early frost. For each plant, SW was determined by weight of the total seed after the cotton ginning processing. To evaluate seed vigor and plumpness, the SI was evaluated for every plant. Descriptive statistical parameters were obtained for each trait.

To assess the relationship between different traits in the reciprocal $F_{2}$ populations, Pearson's correlation coefficients were calculated for traits of individual plants in each population.

\section{Molecular marker genotyping}

Total genomic DNA of the parents and individuals of the two $F_{2}$ segregating populations was extracted from young leaves according to procedures described by Paterson et al. [35]. Since mutual independence is latent premise for MHI calculating, a total of 125 SSRs were randomly selected from the 2316 genome-wide loci according to the genetic distance in the interspecific genetic map previously constructed based on the same parents [36]. These SSR markers were used to genotype the two interspecific reciprocal $F_{2}$ populations. The detail of these markers was presented in Additional file 1: Table S1. The sequences of molecular markers can be obtained from CottonGen (http://www.cottongen.org) [37]. Polymerase chain reaction (PCR) analysis, electrophoresis and silver staining were performed according to procedures described by Lin et al. [38].

\section{Linkage disequilibrium analysis}

Correlations between genotyped markers could lead to deviations in the estimated value of individual molecular hybrid index (MHI). To avoid the correlation, independent assortment between the loci must be validated by Linkage disequilibrium (LD) analysis. LD analysis between all pairs of loci was implemented using the software package TASSEL, which was developed by Edward Buckler's group [39]. LD was estimated by squared allele-frequency correlations $\left(\mathrm{r}^{2}\right)$. According to the definition of LD and calculation formula for $r^{2}$, the bigger $r^{2}$ value is, the degree of correlations or linkage is closer. Correlation was considered to be significant if $\mathrm{r}^{2}>0.2$, and one marker from these pairs was removed. In the end, all markers with $r^{2}<0.2$ were used to estimate individual genomic heterozygosity. After LD analysis, to investigate the correlation between these studied traits and these remaining SSR loci, QTL for these studied traits were searched in these loci from the Cotton QTL Database (http://www2.cottonqtldb.org:8081/index) [40].

\section{Measurement of genomic heterozygosity}

$\mathrm{MHI}$ is an estimate of the proportion of alleles based on molecular markers inherited from alternative parental species in hybrid population [41]. For the interspecific hybrid population from two species, one species is designated as the reference species, the other as the alternative. MHI values range from 0 to 1 , corresponding to pure individuals of alternative and reference species, respectively [41]. MHI is calculated as a measure of individual genomic heterozygosity. Assessment of MHI was performed using the est. $h$ function incorporated in the $\mathrm{R}$ program INTROGRESS [42]. This function renders a maximum likelihood hybrid index estimate for each potentially admixed individual and a $95 \%$ confidence interval for each $[42,43]$.

\section{Analysis of the effects of genomic heterozygosity effect on plant traits}

To assess correlations between genomic heterozygosity and plant traits, Pearson's correlation analysis and one-way analysis of variance (ANOVA) were used to evaluate both populations included in this study. 
First, due to the nature of the MHI, the average value for most heterozygous individuals is 0.5 . Therefore, we divided every population into two groups based on the MHI value of each individual: the members in the first group possessed MHI values greater than 0.5 , and those in the other group had values less than 0.5. Subsequently, Pearson's correlation coefficients were calculated between each trait and the individual heterozygosity value of each plant in every group.

Second, to discover trait differentiation at different genomic heterozygosity levels, the individuals in the two populations were divided into six groups in accordance with individual MHI values, with a step size of 0.05 . The average of the studied traits of each level was calculated for each population, and the statistical significance among groups for each trait was determined by performing separate one-way ANOVAs. Multiple comparisons between each level of genomic heterozygosity were performed using Tukey's multiple comparison tests.

\section{Results}

\section{Hybrid breakdown in interspecific reciprocal $F_{2}$} populations

In total, 142 plants were genotyped simultaneously in each of two interspecific reciprocal $\mathrm{F}_{2}$ populations. Although two plants died after DNA sampling in the (E3) $\mathrm{F}_{2}$ population, the remaining 140 individuals were phenotyped. $\mathrm{PH}, \mathrm{BN}$ and $\mathrm{BrN}$ were investigated for each plant in these two populations. Owing to sterility or flowering delay, only 62 individuals in the (E3) $F_{2}$ population and 51 in the $(3 E) F_{2}$ population were surveyed for two reproductive traits, SW and SI. Frequency distributions of the two interspecific $F_{2}$ populations for $\mathrm{PH}, \mathrm{BrN}, \mathrm{BN}, \mathrm{SW}$ and SI are presented in Fig. 1. Descriptive statistical parameters, such as the mean value, standard deviation, range, skewness, kurtosis and probability of normal distribution for each trait, are shown in Table 1. For both populations, only two traits displayed a normal distribution, $\mathrm{PH}(p=0.20)$ in (3E) $\mathrm{F}_{2}$ and SI $(p=0.69)$ in (E3) $\mathrm{F}_{2}$. The remaining traits displayed non-normal distributions. The data demonstrated that hybrid breakdown may exist in the interspecific reciprocal $F_{2}$ populations particularly with respect to reproductive traits such as infertility and bare seeds.

To investigate possible maternal effects on hybrid breakdown, a two-sample Kolmogorov-Smirnov test was performed between the two populations for the five plant traits. Statistical significance was observed for $\mathrm{PH}$ $(D=0.2173, p=0.0026)$ and SI $(D=0.3577, p=0.0021)$, suggesting that the maternal cytoplasmic environment could have significant effects on PH and SI.

Correlations between the vegetative and reproductive traits in the two interspecific reciprocal $\mathrm{F}_{2}$ populations are listed in Table 2. In the (E3) $F_{2}$ population, significantly positive correlations were observed between $\mathrm{PH}$ and $\mathrm{BrN}(r=0.42, p=0)$ as well as between BN and SW $(r=0.40, p=0)$. In the (3E) $\mathrm{F}_{2}$ population, significantly positive correlations were observed between $\mathrm{PH}$ and SW $(r=0.28, p=0.04), \mathrm{PH}$ and BN $(r=0.38, p=0), \mathrm{PH}$ and $\operatorname{BrN}(r=0.37, p=0), \mathrm{BN}$ and $\operatorname{BrN}(r=0.34, p=0)$, and $\mathrm{BN}$ and SW $(r=0.64, p=0)$. These data implied that the correlations among the vegetative and reproductive traits in the (E3) $F_{2}$ population were nonsignificant; however, this was not the case in the (3E) $F_{2}$ population.

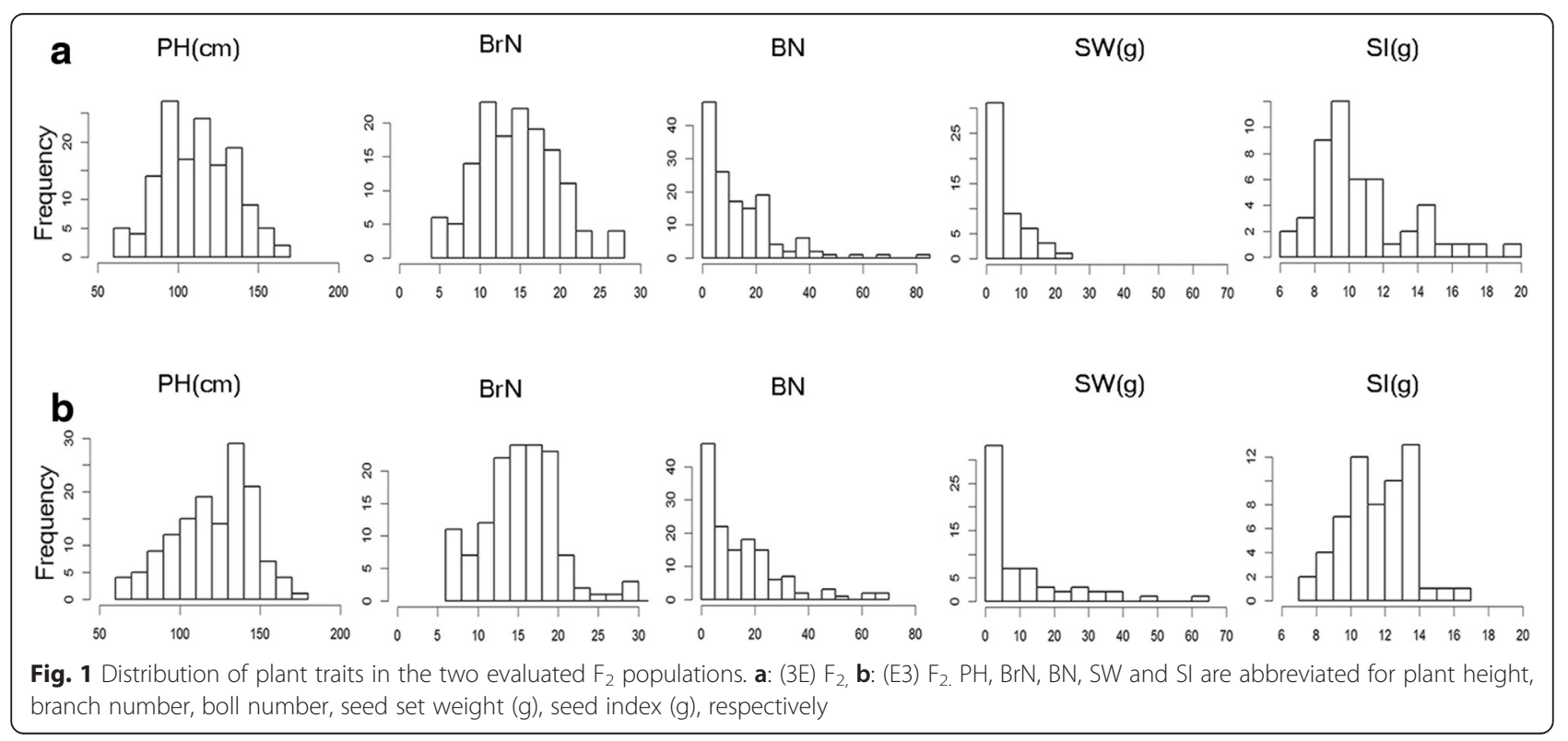


Table 1 Descriptive statistics of plant traits in the two evaluated $F_{2}$ populations

\begin{tabular}{llllccccc}
\hline Population & Trait & Size & Range & Mean \pm S.D. & SKE. & KUR & NT.W & NT.p \\
\hline (3E) $F_{2}$ & Plant height $(\mathrm{cm})$ & 142 & $60.00-165.00$ & $114.32 \pm 22.21$ & 0.05 & -0.48 & 0.99 & 0.20 \\
& Branch number & 142 & $5.00-28.00$ & $15.06 \pm 4.88$ & 0.25 & -0.24 & 0.98 & 0.12 \\
& Boll number & 142 & $0.00-81.00$ & $14.16 \pm 13.66$ & 1.78 & 4.55 & 0.84 & 0.00 \\
& Seed set weight (g) & 51 & $0.32-20.77$ & $5.86 \pm 5.15$ & 1.26 & 0.52 & 0.83 & 0.00 \\
& Seed index (g) & 50 & $6.10-19.58$ & $10.75 \pm 2.86$ & 1.12 & 0.82 & 0.90 & 0.00 \\
$\left(\right.$ E3) $F_{2}$ & Plant height (cm) & 140 & $60.00-180.00$ & $123.75 \pm 25.18$ & -0.35 & -0.49 & 0.98 & 0.02 \\
& Branch number & 138 & $6.00-38.00$ & $15.95 \pm 5.01$ & 0.77 & 2.35 & 0.95 & 0.00 \\
& Boll number & 140 & $0.00-69.00$ & $14.82 \pm 14.74$ & 1.50 & 2.52 & 0.85 & 0.00 \\
& Seed set weight (g) & 62 & $0.32-63.44$ & $10.47 \pm 13.23$ & 1.87 & 3.32 & 0.74 & 0.00 \\
& Seed index (g) & 60 & $7.14-16.64$ & $11.47 \pm 1.96$ & 0.03 & -0.33 & 0.99 & 0.69 \\
& & & & & &
\end{tabular}

Loci independence check by linkage disequilibrium analysis

To avoid the correlation between paired loci, mutual independence between the loci must be validated by LD analysis. The $\mathrm{r}^{2}$ and $\mathrm{p}$ values between all loci pairs based on $\mathrm{LD}$ testing and the $\mathrm{r}^{2}$ distributions of the two $\mathrm{F}_{2}$ populations are presented in Fig. 2. After pre-processing, 87 loci were shared in the two interspecific reciprocal $\mathrm{F}_{2}$ populations. Four locus pairs showed significant LD. Following the above, one marker from each locus pair was removed from the dataset. To test the substitution effect produced by this method, molecular hybrid indices for each marker from these pairs were calculated (Additional file 2: Tables S2 and Additional file 3: Table S3). To determine the statistical significance of the differences among these MHIs, a two-sample Kolmogorov-Smirnov test was performed between each in the two populations. The results showed that no statistical significance was observed between MHIs after replacement in the (3E) $F_{2}$ population and (E3) $\mathrm{F}_{2}$ population. That is, if NAU3130 was replaced with NAU3010a $(D=0.0704, p=0.87)$, NAU5345 was replaced with NBRI_HQ526876 $(D=0.0845$, $p=0.69)$, or the two markers were replaced together $(D=0.0845, p=0.69)$ in the $(3 \mathrm{E}) \mathrm{F}_{2}$ population, there was no statistically significant difference. Similarly, in the (E3) $\mathrm{F}_{2}$ population, if HAU2313 was replaced with HAU4369 $(D=0.0786, p=0.78)$, HAU1577 was replaced with MON-CGR6528 $(D=0.0571, p=0.97)$ or the two markers were replaced together $(D=0.0571, p=0.97)$, there was no significant difference. These results indicated that this method was in accordance with our expectations. Meanwhile, to investigate correlation between the studied traits and these remaining SSR loci, QTL correlated to the studied traits were searched from Cotton QTL Database (http://www2.cottonqtldb.org:8081/index) [40], the results (Additional file 4: Tables S4) showed no markers were correlated to the studied traits. The remaining 83 neutral loci in the two populations were used for further analysis.

\section{Distribution of individual genomic heterozygosity}

Individual genomic composition patterns were found in the 83 SSR loci, as shown in Fig. 3 (a, b). Allele distribution from E22 and 3-79 occurred randomly both among the individuals in the two populations and among different genomic regions in individuals.

Individual genomic heterozygosity was estimated using the 83 SSR loci common to the two populations. Frequency distributions of the MHIs in the two interspecific reciprocal $F_{2}$ populations are presented in Fig 3 (c, d).

In the $(3 \mathrm{E}) \mathrm{F}_{2}$ population, the $\mathrm{MHI}$ ranged from 0.39 to 0.62 with a mean and standard deviation of 0.497 and 0.040, respectively. The Shapiro-Wilk test revealed that the distribution of the MHI was normal ( $W=0.9906$, $p=0.4645)$. In the (E3) $\mathrm{F}_{2}$ population, the MHI ranged from 0.21 to 0.71 with a mean and standard

Table 2 Pearson's correlation coefficients between plant traits in the two evaluated populations

\begin{tabular}{|c|c|c|c|c|c|}
\hline & Plant height $(\mathrm{cm})$ & Branch number & Boll number & Seed set weight (g) & Seed index (g) \\
\hline Plant height (cm) & - & $0.42^{* *}$ & 0.12 & 0.14 & 0.16 \\
\hline Branch number & $0.37^{* *}$ & - & 0.15 & 0.05 & -0.13 \\
\hline Boll number & $0.38^{* *}$ & $0.34^{* *}$ & - & $0.40^{* *}$ & -0.07 \\
\hline Seed set weight (g) & $0.28^{*}$ & 0.27 & $0.64^{* *}$ & - & 0.11 \\
\hline Seed index (g) & -0.03 & -0.03 & -0.03 & 0.07 & - \\
\hline
\end{tabular}

Entries above the diagonal represent the correlation coefficient for (E3) $F_{2}$; entries below the diagonal represent the correlation coefficient for (3E) $\mathrm{F}_{2}{ }^{* *} p<0.01,{ }^{*} p<0.05$ 

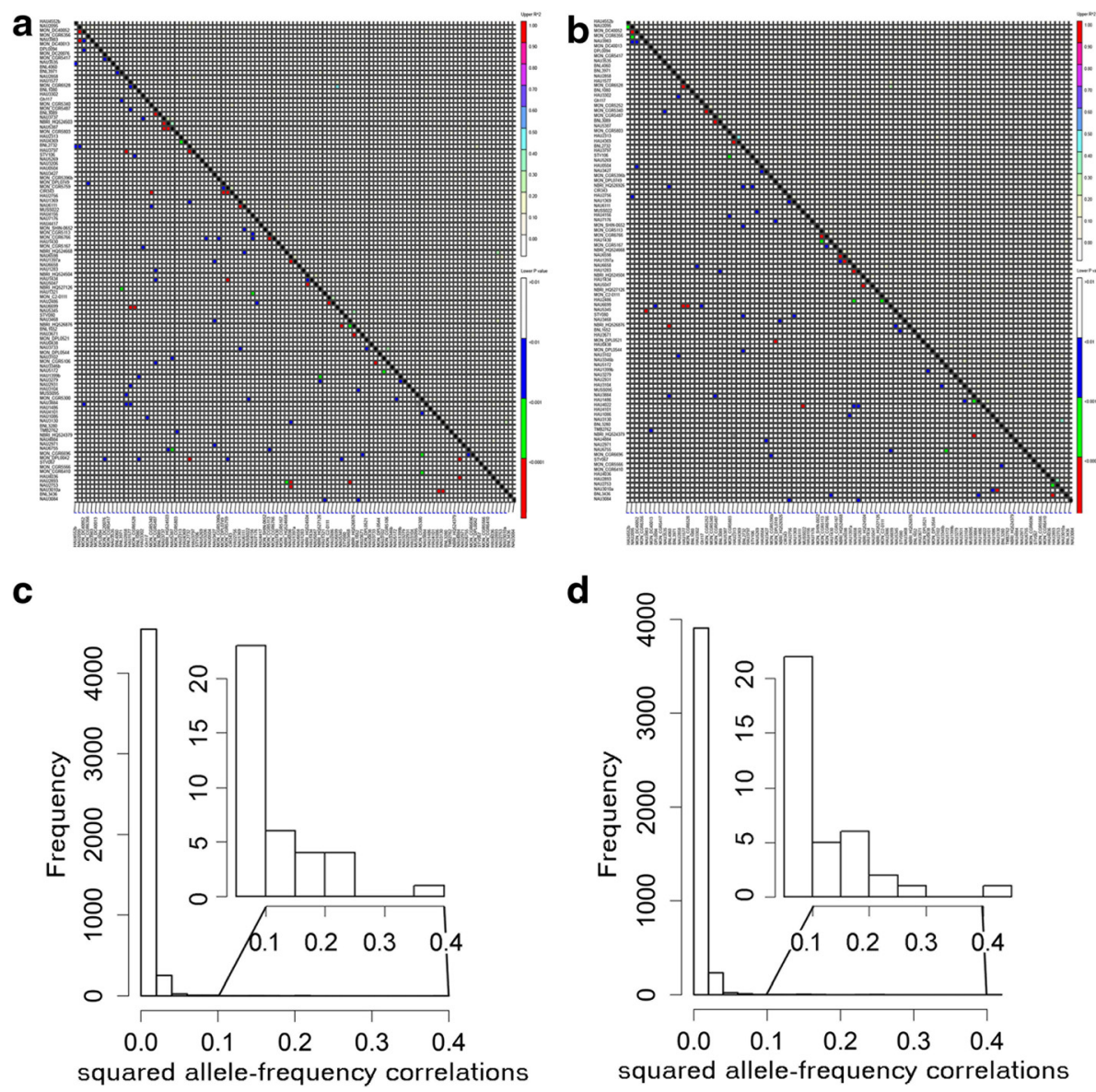

Fig. 2 Pairwise linkage disequilibrium between all markers in both populations. The $r^{2}$ and $p$-values from linkage disequilibrium testing of the (3E) $F_{2}(\mathbf{a})$ and $(E 3) F_{2}(\mathbf{b})$ are above the diagonal and below the diagonal, respectively. The $r^{2}$ distributions in (3E) $F_{2}(\mathbf{c})$ and $(E 3) F_{2}(\mathbf{d})$ are shown

deviation of 0.480 and 0.080 , respectively. The MHI distribution was assessed for normality using the Shapiro-Wilk test, and an abnormal distribution was noted $(W=0.8988$, $p=2.713 \mathrm{e}-08$ ).

The average MHI in each population was less than the expected value (0.5), indicating that the genomic composition from 3-79 was greater than that from E22, which suggests that the alleles from 3-79 may have a stronger selective advantage.

To investigate the statistical significance of the difference in the MHIs, a two-sample Kolmogorov-Smirnov test was performed on the two populations, which indicated that the two distributions were not drawn randomly from the same population $(D=0.1732, p=0.0290)$. This result suggests that the maternal cytoplasmic environment may have significant effects on individual genomic heterozygosity.

\section{Relationship between genomic heterozygosity and hybrid breakdown}

Pearson's correlation analysis and one-way ANOVA were performed to detect the relationship between genomic heterozygosity and hybrid breakdown. Two groups were divided from each population according to individual MHIs; one group had an MHI greater than 0.5, and the other group did not.

The resulting Pearson's correlation coefficients in the two populations are presented in Table 3. Significant correlations between $\mathrm{MHI}$ and $\mathrm{BN}(r=0.54, p=0)$ as well as between MHI and SW $(r=0.70, p=0)$ were observed in the group $(\mathrm{MHI}<0.5)$ from the $(\mathrm{E} 3) \mathrm{F}_{2}$ population. However, the other $p$-values of the Pearson's correlation coefficients were greater than 0.05 , demonstrating that no significant correlations were found. In other words, hybrid breakdown relative to the other traits in the interspecific reciprocal $F_{2}$ populations was unrelated to genomic heterozygosity.

According to individual MHIs, six groups were defined in the two populations, with levels 1 to level 6 including all observed values. The number of individuals and the mean and MHI range of each of the six levels are presented in Table 4. These results are consistent with the expectations of the $\mathrm{F}_{2}$ population, namely, $62.14 \%$ of individuals in the (E3) $\mathrm{F}_{2}$ population and $80.28 \%$ of individuals in the (3E) $\mathrm{F}_{2}$ population were noted in levels 3 and 4, with MHIs ranging from 0.45 to 0.55 . 

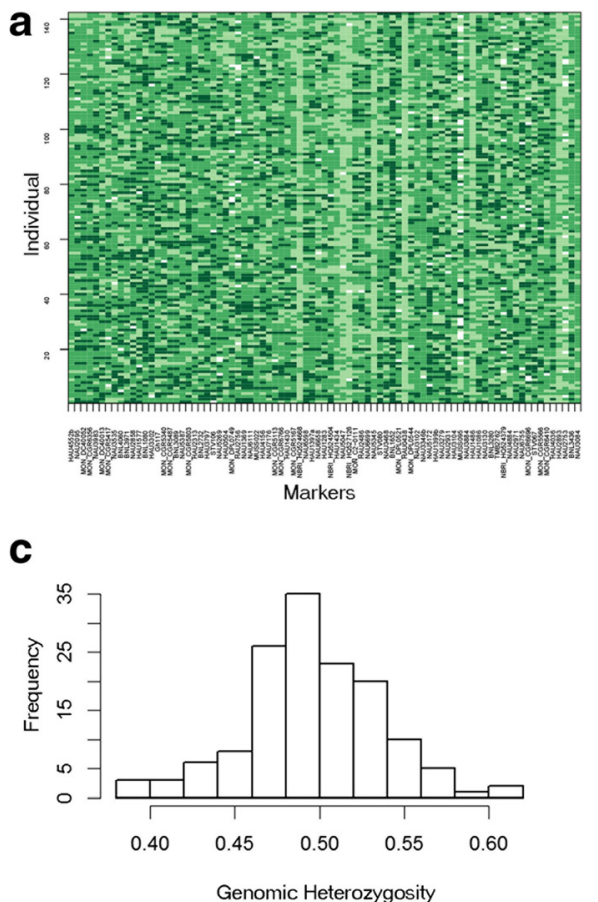

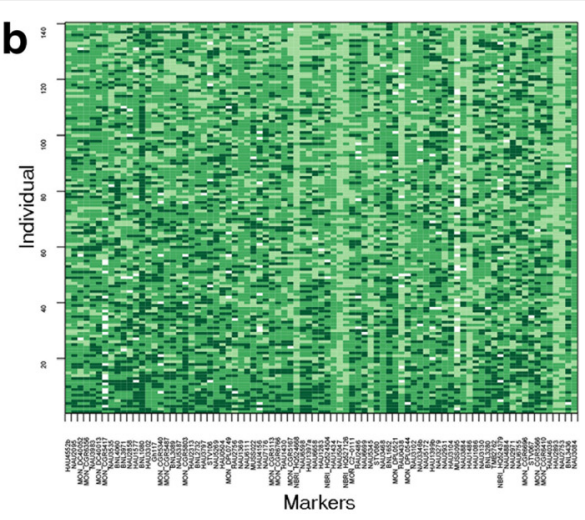

d

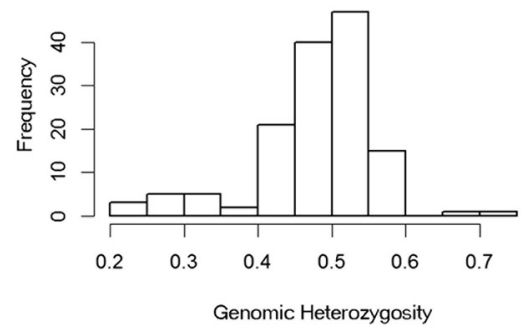

Fig. 3 The constructed specifications for all markers and individuals in (3E) $F_{2}(\mathbf{a})$ and (E3) $F_{2}(\mathbf{b})$ and the frequency distributions of $M H I s$ in (3E) $F_{2}$ (c) and (E3) $F_{2}$ (d). Each rectangle denotes an individual's genotype at a given locus. Colours are arranged from darker green, indicating E22 homozygotes, to green, indicating heterozygote genotypes between E22 and 3-79, to light green, indicating 3-79 homozygotes. White blocks indicate missing data

The mean trait performance at each level of genomic heterozygosity is presented in Table 5. One-way ANOVA was used to determine group differences between these two populations. No significant differences were observed between plant traits and individual genomic heterozygosity levels in the (3E) $F_{2}$ population. Nevertheless, significant differences were observed between $\mathrm{BN}\left(F_{5}, 134=\right.$ 4.925, $p=0.00036)$ and SW $\left(F_{5,55}=10.59, p=3.72 \mathrm{e}-07\right)$ and individual genomic heterozygosity level in the (E3) $\mathrm{F}_{2}$ population. These results reconfirmed that plant vegetative traits, i.e., $\mathrm{PH}$ and $\mathrm{BrN}$, have no correlation with individual genomic heterozygosity; however, genomic heterozygosity may affect reproductive traits, such as $\mathrm{BN}$ and $\mathrm{SW}$.

Table 3 Pearson's correlation coefficients between plant traits and $\mathrm{MHI}$ in both populations

\begin{tabular}{|c|c|c|c|c|c|c|c|c|}
\hline \multirow[t]{3}{*}{ Correlation } & \multicolumn{4}{|l|}{ (3E) $F_{2}$} & \multicolumn{4}{|l|}{$(\mathrm{E} 3) \mathrm{F}_{2}$} \\
\hline & \multicolumn{2}{|c|}{$\mathrm{MHI}<0.5$} & \multicolumn{2}{|c|}{$\mathrm{MHI}>0.5$} & \multicolumn{2}{|c|}{$\mathrm{MHI}<0.5$} & \multicolumn{2}{|c|}{$\mathrm{MHI}>0.5$} \\
\hline & $r$ & $p$ & $r$ & $p$ & $r$ & $p$ & $R$ & $p$ \\
\hline Plant height $(\mathrm{cm})$ & -0.12 & 1 & 0.09 & 1 & -0.09 & 1 & 0.11 & 1 \\
\hline Branch number & -0.07 & 1 & 0.01 & 1 & -0.13 & 1 & 0.2 & 1 \\
\hline Boll number & 0.05 & 1 & 0.02 & 1 & -0.54 & 0 & 0.18 & 1 \\
\hline Seed set weight (g) & -0.12 & 1 & 0.13 & 1 & -0.7 & 0 & 0.17 & 1 \\
\hline Seed index (g) & 0.37 & 0.83 & -0.09 & 1 & -0.2 & 1 & -0.03 & 1 \\
\hline
\end{tabular}

The results from the pairwise comparisons between $\mathrm{BN}$ and $\mathrm{SW}$ in the (E3) $\mathrm{F}_{2}$ population are shown in Table 5 and Fig. 4. Significant differences for BN and SW were detected between level 1 and levels 2, 3, 4, as well as between level 1 and levels 2, 3, 4, 5. No significant differences were detected among the other pairwise comparisons.

According to the definition of MHI, higher MHI corresponds to higher levels of individual genomic heterozygosity, when MHI values are less than 0.5. Through comparative analysis of $\mathrm{BN}$ and $\mathrm{SW}$ at different levels in the (E3) $F_{2}$ population, we conclude that increased genomic heterozygosity indicates lower $\mathrm{BN}$ and $\mathrm{SW}$ values

Table $4 \mathrm{MHI}$ range, mean and observation number (\%) for the six genomic heterozygosity levels in the two evaluated populations

\begin{tabular}{|c|c|c|c|c|c|c|}
\hline \multirow[t]{2}{*}{ Level } & \multicolumn{3}{|c|}{ (3E) $F_{2}$} & \multicolumn{3}{|c|}{ (E3) $F_{2}$} \\
\hline & Size & Mean & Percentage (\%) & Size & Mean & Percentage (\%) \\
\hline$<0.4$ & 3 & 0.40 & 2.11 & 15 & 0.30 & 10.71 \\
\hline $0.4 \sim 0.45$ & 12 & 0.43 & 8.45 & 21 & 0.43 & 15.00 \\
\hline $0.45 \sim 0.5$ & 62 & 0.48 & 43.66 & 37 & 0.48 & 26.43 \\
\hline $0.5 \sim 0.55$ & 52 & 0.52 & 36.62 & 50 & 0.52 & 35.71 \\
\hline $0.55 \sim 0.6$ & 11 & 0.56 & 7.75 & 15 & 0.57 & 10.71 \\
\hline$>0.6$ & 2 & 0.61 & 1.41 & 2 & 0.69 & 1.43 \\
\hline
\end{tabular}


Table 5 Comparisons across MHI levels in trait performances from both populations

\begin{tabular}{|c|c|c|c|c|c|c|c|c|c|c|}
\hline \multirow[t]{2}{*}{ Level } & \multicolumn{5}{|l|}{ (3E) $F_{2}$} & \multicolumn{5}{|l|}{ (E3) $F_{2}$} \\
\hline & $\mathrm{PH}(\mathrm{cm})$ & $\mathrm{BrN}$ & $\mathrm{BN}$ & $\mathrm{SW}(\mathrm{g})$ & $\mathrm{SI}(\mathrm{g})$ & $\mathrm{PH}(\mathrm{cm})$ & $\mathrm{BrN}$ & $\mathrm{BN}$ & SW(g) & $\mathrm{SI}(\mathrm{g})$ \\
\hline$<0.4$ & 108.00 & 13.33 & 4.33 & 1.24 & 6.10 & 118.60 & 16.47 & $29.80 \mathrm{~b}$ & 29.73 b & 11.93 \\
\hline $0.4 \sim 0.45$ & 118.25 & 15.83 & 12.25 & 9.39 & 8.86 & 123.00 & 16.71 & $14.24 \mathrm{a}$ & 6.62 a & 11.13 \\
\hline $0.45 \sim 0.5$ & 109.50 & 14.37 & 12.27 & 6.08 & 10.09 & 116.95 & 15.58 & $9.05 \mathrm{a}$ & $3.61 \mathrm{a}$ & 11.18 \\
\hline $0.5 \sim 0.55$ & 117.35 & 15.77 & 16.38 & 5.35 & 11.59 & 129.52 & 15.59 & $14.36 \mathrm{a}$ & 6.86 a & 11.69 \\
\hline $0.55 \sim 0.6$ & 127.73 & 16.27 & 20.73 & 6.97 & 10.93 & 125.67 & 16.07 & $15.67 \mathrm{ab}$ & $8.18 \mathrm{a}$ & 11.1 \\
\hline$>0.6$ & 97.00 & 9.50 & 5.00 & - & - & 137.50 & 18.50 & $20.50 a b$ & $13.14 a b$ & 12.43 \\
\hline
\end{tabular}

The presented values in the table are the mean of different levels of the studied traits. Statistical significance in each group was analysed by one-way ANOVA with multiple comparisons being made by Tukey's test. Significant differences between level groups are indicated by letters $(p<0.05)$. $\mathrm{PH}, \mathrm{BrN}$, BN, $\mathrm{SW}$ and $\mathrm{SI}$ are abbreviated for plant height, branch number, boll number, seed set weight $(\mathrm{g})$, seed index (g), respectively

when MHI is less than 0.5. These results are consistent with our Pearson's correlation analysis, reconfirming the negative correlations between $\mathrm{MHI}$ and $\mathrm{BN}$ and between MHI and SW in the (E3) $F_{2}$ population. As such, hybrid breakdown in $\mathrm{BN}$ and $\mathrm{SW}$ exhibited positive correlations with genomic heterozygosity. However, this was not valid in the $(3 \mathrm{E}) \mathrm{F}_{2}$ population.

\section{Discussion}

\section{A novel approach for assessing genomic heterozygosity}

Heterozygosity-fitness correlations have been used to study the relationship between genomic heterozygosity and fitness-related traits at an individual level in natural hybrid populations of a variety of organisms [8-12]. Nonetheless, associations of marker loci have been ignored in previous studies of genomic heterozygosity, which could give rise to an estimate bias if such associations are true. As an extreme example, calculated genomic heterozygosity only represents chromosome heterozygosity, in cases where all markers are located on the same chromosome.

In the current study, the loci for MHI calculating were randomly selected from the 2316 genome-wide loci cotton interspecific genetic map [34], the correlation between paired loci were checked by LD analysis, independent assortment between the loci must be validated. Genomewide LD patterns in hybrid populations have been studied in a few organisms [44-46]. These studies suggested that LD analysis can be used to estimate correlation among marker loci in hybrid populations.

In our study, a total of 83 common loci were determined through marker-checking, which were subsequently used to assess genomic heterozygosity. To the best of our knowledge, this is an improved approach for assessing genomic heterozygosity, which could be applicable to many species that are currently the focus of heterozygosity-fitness correlation research in evolution and ecology. It could also provide new insights for assessing general genome-wide heterozygosity.

\section{Hybrid breakdown in interspecific reciprocal $F_{2}$ cotton populations}

Previous works have documented hybrid breakdown in the $\mathrm{F}_{2}$ and later generations of interspecific hybrids between $G$. hirsutum and G. barbadense [33, 47-49]. For example,
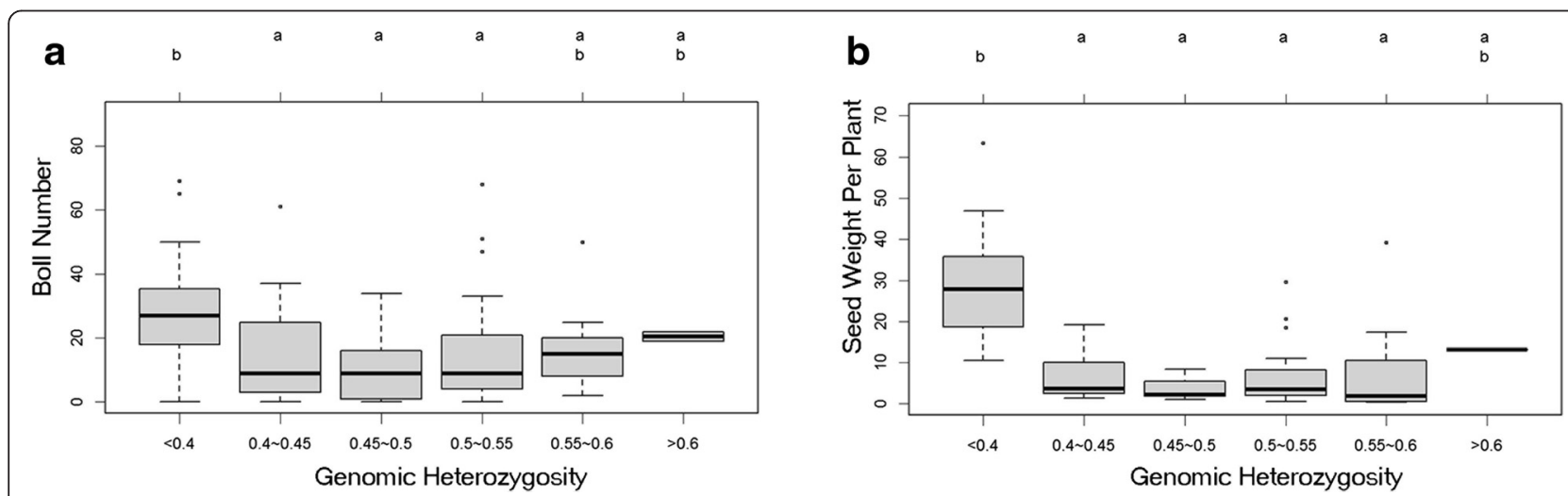

Fig. 4 Boxplot of plant traits across groups of genomic heterozygosity for boll number (a) and seed set weight (b). Multiple group comparisons were performed using one-way ANOVA followed by Tukey's multiple comparison tests. The bottoms and tops of the boxes represent 25 and $75 \%$, respectively; the bands near the middle indicate the median. The ends of the whiskers represent 10 and $90 \%$. The letters above the boxes indicate significant differences between the groups. Groups that do not share a letter differ significantly $(p<0.05)$ 
Jiang et al. [47] reported that multilocus epistatic interactions affected gene transmission in an interspecific population of polyploid Gossypium. However, such studies have not focused on the effect of hetero-species cytoplasm on hybrid breakdown in cotton interspecific reciprocal populations. In the current study, we assessed the effect of heterospecies cytoplasm by comparing plant trait performance in cotton interspecific reciprocal $F_{2}$ populations. The cytoplasmic environment had significant effects on $\mathrm{PH}$ and SI, and this study therefore suggested that the cytoplasmic environment might play an important role in hybrid breakdown in cotton interspecific reciprocal $\mathrm{F}_{2}$ populations.

\section{Effect of genomic heterozygosity on hybrid breakdown}

Relationships between individual genomic-wide general heterozygosity and phenotypic traits have been studied both in both animals and plants $[8,9,24,29,50]$. According to previous studies, the relationships between individual genome-wide general heterozygosity and traits were very sophisticated and might differ with respect to sampling species and assessment procedure. For example, significant positive effects between standardized multilocus heterozygosity as calculated by neutral loci and adult survival were found in blue tits (Cyanistes caeruleus) [11]. Abrahamsson et al. [10] found that standardized multilocus heterozygosity in mother trees had a significant negative effect on mean offspring height in an inbred Scots pine population. Savolainen and Hedrick [8] had reported that no evidence of an association was found between vegetative and reproductive fitness-related traits and heterozygosity in P. sylvestris.

The majority of current studies on heterozygosity-fitness correlations have focused on natural populations in hybrid zones. In the current study, we assessed relationships between genomic heterozygosity and hybrid traits in two artificial hybrid populations derived from a reciprocal cross between G. hirsutum and G. barbadense. In the (3E) $\mathrm{F}_{2}$ population, no correlations were observed between the measured plant traits and genomic heterozygosity, indicating that hybrid breakdown may be not linked with genomic heterozygosity. On the other hand, correlations were only observed between SI, BN and genomic heterozygosity in the (E3) $F_{2}$ population. These findings illustrated that cytoplasms from different species might significantly impact such relationships.

Our results also indicated that hybrid performance in the G. barbadense cytoplasmic background might have no correlation with genomic heterozygosity, regardless of whether vegetative traits or reproductive traits were being assessed; however, in the G. hirsutum cytoplasmic background, the reproductive traits SI and BN were associated with genomic heterozygosity. These results suggested that the G. barbadense cytoplasm background could exhibit better compatibility compared with that of G. hirsutum. Our results may therefore offer new insights into hybrid breakdown in allotetraploid cotton interspecific hybrids and may provide a fresh perspective on interspecific hybridization for the genetic improvement of cotton.

\section{Conclusions}

To investigate the correlations between genomic heterozygosity and vegetative and reproductive traits in allotetraploid cotton, two reciprocal $\mathrm{F}_{2}$ populations were developed using G. hirsutum cv. Emian 22 and G. barbadense acc. 379 as parents. A total of 125 SSR markers were evaluated by marker pair correlations using linkage disequilibrium analysis, and 83 common loci were used to assess the extent of genomic heterozygosity. The vegetative traits $(\mathrm{PH}$ and $\mathrm{BrN})$ and reproductive traits $(\mathrm{BN}, \mathrm{SW}$ and $\mathrm{SI})$ were investigated in the in allotetraploid cotton interspecific populations, and hybrid breakdown was found extensively in the two interspecific $\mathrm{F}_{2}$ populations particularly on the reproductive traits because of the infertility and the bare seeds. The only relationships between hybrid breakdown and heterozygosity was observed for $\mathrm{SI}$ and $\mathrm{BN}$ in the (E3) $F_{2}$ population. The maternal cytoplasmic environment may have a significant effect on genomic heterozygosity and on correlations between heterozygosity and reproductive traits.

\section{Ethics (and consent to participate)}

Not applicable.

\section{Consent to publish}

Not applicable.

\section{Availability of data and materials}

All relevant data are available within the manuscript and its additional files.

\section{Additional files}

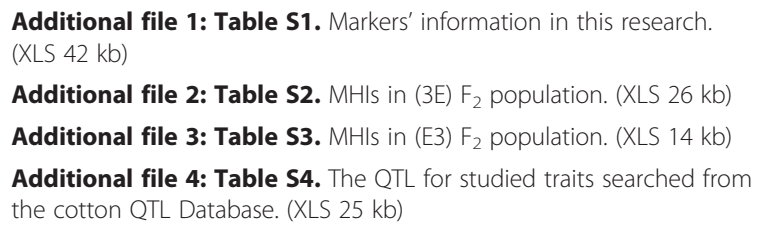

\section{Abbreviations}

ANOVA: analysis of variance; ATP: adenosine triphosphate; BDM

incompatibilities: Bateson-Dobzhansky-Muller (BDM) incompatibilities;

BN: boll number; BrN: branch number; E22: Emian 22; LD: linkage

disequilibrium; $\mathrm{MHI}$ : molecular hybrid index; $\mathrm{PH}$ : plant height;

QTL: quantitative trait loci; SI: seed index; SNPs: single-nucleotide

polymorphisms; SSRs: simple sequence repeats; SW: seed set weight.

Competing interests

The authors declare that they have no competing interests. 


\section{Authors' contributions}

BSD carried out molecular marker genotyping, performed analyses of the genetic data and drafted the article. HLG and CH aided in genotyping a portion of the molecular markers. XLZ and ZXL designed the study and oversaw all of lab work and analyses. All authors read and approved the final manuscript.

\section{Acknowledgments}

Professor Keith Lindsey from Durham University should also be acknowledged for his help on the manuscript writing.

\section{Funding}

This work was financially supported by the National Science Foundation of China (No. 31171593).

\section{Received: 19 October 2015 Accepted: 7 April 2016}

\section{Published online: 12 April 2016}

\section{References}

1. Ellegren $H$, Smeds L, Burri R, Olason PI, Backstrom N, Kawakami T, Kunstner A, Makinen H, Nadachowska-Brzyska K, Qvarnstrom A. The genomic landscape of species divergence in Ficedula flycatchers. Nature. 2012; 491(7426):756-60

2. Oneal E, Knowles LL. Ecological selection as the cause and sexual differentiation as the consequence of species divergence? Proc R Soc Lond B Biol Sci. 2013;280(1750):2236.

3. Matsubara K, Yamamoto E, Mizobuchi R, Yonemaru J, Yamamoto T, Kato H, Yano M. Hybrid breakdown caused by epistasis-based recessive incompatibility in a cross of rice (Oryza sativa L.). J Hered. 2015;106(1):113-22.

4. Burton RS, Pereira RJ, Barreto FS. Cytonuclear genomic interactions and hybrid breakdown. Annu Rev Ecol Evol Syst. 2013:44(1):281-302.

5. Yamamoto E, Takashi T, Morinaka Y, Lin S, Wu J, Matsumoto T, Kitano H, Matsuoka M, Ashikari M. Gain of deleterious function causes an autoimmune response and Bateson-Dobzhansky-Muller incompatibility in rice. Mol Genet Genomics. 2010;283(4):305-15.

6. Yamamoto E, Takashi T, Morinaka Y, Lin S, Kitano H, Matsuoka M, Ashikari M. Interaction of two recessive genes, $h b d 2$ and $h b d 3$, induces hybrid breakdown in rice. Theor Appl Genet. 2007:115(2):187-94.

7. Gibson JD, Niehuis O, Peirson BR, Cash El, Gadau J. Genetic and developmental basis of $\mathrm{F}_{2}$ hybrid breakdown in Nasonia parasitoid wasps. Evolution. 2013;67(7):2124-32

8. Savolainen O, Hedrick P. Heterozygosity and fitness: no association in Scots pine. Genetics. 1995;140(2):755-66.

9. Vranckx G, Jacquemyn H, Mergeay J, Cox K, Janssens P, Gielen BAS, Muys B, Honnay $O$. The effect of drought stress on heterozygosity-fitness correlations in pedunculate oak (Quercus robur). Ann Bot. 2014;113(6):1057-69.

10. Abrahamsson S, Ahlinder J, Waldmann P, Garcia-Gil MR. Maternal heterozygosity and progeny fitness association in an inbred Scots pine population. Genetica. 2013:141(1-3):41-50.

11. Olano-Marin J, Mueller JC, Kempenaers B. Heterozygosity and survival in blue tits (Cyanistes caeruleus): contrasting effects of presumably functional and neutral loci. Mol Ecol. 2011;20(19):4028-41.

12. Harrison XA, Bearhop $S$, Inger $R$, Colhoun $K$, Gudmundsson GA, Hodgson D, McElwaine G, Tregenza T. Heterozygosity-fitness correlations in a migratory bird: an analysis of inbreeding and single-locus effects. Mol Ecol. 2011 20(22):4786-95.

13. Ouyang $Y$, Zhang Q. Understanding reproductive isolation based on the rice model. Annu Rev Plant Biol. 2013;64:111-35.

14. Edmands S, Northrup SL, Hwang AS. Maladapted gene complexes within populations of the intertidal copepod Tigriopus californicus. Evolution. 2009; 63(8):2184-92

15. Kubo T, Yoshimura A. Epistasis underlying female sterility detected in hybrid breakdown in a Japonica-Indica cross of rice (Oryza sativa L.). Theor App Genet. 2005;110(2):346-55.

16. Bikard D, Patel D, Le Mette C, Giorgi V, Camilleri C, Bennett MJ, Loudet O. Divergent evolution of duplicate genes leads to genetic incompatibilities within A. thaliana. Science. 2009:323(5914):623-6.

17. Ellison CK, Burton RS. Interpopulation hybrid breakdown maps to the mitochondrial genome. Evolution. 2008:62(3):631-8.

18. Clarke GM. The genetic basis of developmental stability. I. Relationships between stability, heterozygosity and genomic coadaptation. Genetica. 1993;89(1-3):15-23.
19. Dobzhansky T, Pavlovsky O. Interracial hybridization and breakdown of coadapted gene complexes in Drosophila paulistorum and Drosophila willistoni. Proc Natl Acad Sci U S A. 1958;44(6):622-9.

20. Wetzel DP, Stewart IR, Westneat DF. Heterozygosity predicts clutch and egg size but not plasticity in a house sparrow population with no evidence of inbreeding. Mol Ecol. 2012;21(2):406-20.

21. Hamilton JA, Lexer C, Aitken SN. Genomic and phenotypic architecture of a spruce hybrid zone (Picea sitchensis P. glauca). Mol Ecol. 2013;22(3):827-41.

22. Aparicio JM, Ortego J, Cordero PJ. What should we weigh to estimate heterozygosity, alleles or loci? Mol Ecol. 2006;15(14):4659-65.

23. Balloux F, Amos W, Coulson T. Does heterozygosity estimate inbreeding in real populations? Mol Ecol. 2004;13(10):3021-31.

24. Szulkin M, Bierne N, David P. Heterozygosity-fitness correlations: a time for reappraisal. Evolution. 2010;64(5):1202-17.

25. Jose Ruiz-Lopez M, Ganan N, Antonio Godoy J, Del Olmo A, Garde J, Espeso G, Vargas A, Martinez F, Roldan ERS, Gomendio M. Heterozygosity-Fitness correlations and inbreeding depression in two critically endangered mammals. Conserv Biol. 2012;26(6):1121-9.

26. Voegeli B, Saladin V, Wegmann M, Richner H. Parasites as mediators of heterozygosity-fitness correlations in the Great Tit (Parus major). J Evol Biol. 2012; 25(3):584-90.

27. Miller JM, Malenfant RM, David P, Davis CS, Poissant J, Hogg JT, FestaBianchet $\mathrm{M}$, Coltman DW. Estimating genome-wide heterozygosity: effects of demographic history and marker type. Heredity. 2014;112(3):240-7.

28. Fitzpatrick BM. Estimating ancestry and heterozygosity of hybrids using molecular markers. BMC Evol Biol. 2012;12:131.

29. Chapman JR, Nakagawa S, Coltman DW, Slate J, Sheldon BC. A quantitative review of heterozygosity-fitness correlations in animal populations. Mol Ecol. 2009;18(13):2746-65.

30. Paterson AH. Genetics and genomics of cotton, vol. 3. New York: SpringerVerlag; 2009.

31. Zhang J, Percy RG, McCarty Jr JC. Introgression genetics and breeding between Upland and Pima cotton: a review. Euphytica. 2014;198(1):1-12.

32. Kohel R, Endrizzi J, White T. An evaluation of Gossypium barbadense L. chromosomes 6 and 17 in the G. hirsutum L. genome. Crop Sci. 1977;17(3):404-6.

33. Stephens S. The cytogenetics of speciation in Gossypium. I. Selective elimination of the donor parent genotype in interspecific backcrosses. Genetics. 1949:34(5):627.

34. Kohel RJ. Genetic nomenclature in cotton. J Hered. 1973;64(5):291-5

35. Paterson $\mathrm{AH}$, Brubaker $\mathrm{CL}$, Wendel JF. A rapid method for extraction of cotton (Gossypium spp.) genomic DNA suitable for RFLP or PCR analysis. Plant Mol Biol Rep. 1993;11(2):122-7.

36. Yu Y, Yuan DJ, Liang SG, Li XM, Wang XQ, Lin ZX, Zhang XL. Genome structure of cotton revealed by a genome-wide SSR genetic map constructed from a $\mathrm{BC}_{1}$ population between Gossypium hirsutum and G. barbadense. BMC Genomics. 2011;12:15.

37. Yu J, Jung $S$, Cheng CH, Ficklin SP, Lee T, Zheng P, Jones D, Percy RG, Main D. CottonGen: a genomics, genetics and breeding database for cotton research. Nucleic Acids Res. 2014:42(Database issue):D1229-36.

38. Lin ZX, He D, Zhang XL, Nie Y, Guo X, Feng C, Stewart JM. Linkage map construction and mapping QTL for cotton fibre quality using SRAP, SSR and RAPD. Plant Breed. 2005;124(2):180-7.

39. Bradbury PJ, Zhang Z, Kroon DE, Casstevens TM, Ramdoss Y, Buckler ES TASSEL: software for association mapping of complex traits in diverse samples. Bioinformatics. 2007:23(19):2633-5.

40. Said Jl, Knapka JA, Song MZ, Zhang JF. Cotton QTLdb: a cotton QTL database for QTL analysis, visualization, and comparison between Gossypium hirsutum and G. hirsutum × G. barbadense populations. Mol Genet Genomics. 2015;290(4):1615-25.

41. Buerkle CA. Maximum-likelihood estimation of a hybrid index based on molecular markers. Mol Ecol Notes. 2005:5(3):684-7.

42. Gompert Z, Buerkle CA. INTROGRESS: a software package for mapping components of isolation in hybrids. Mol Ecol Resour. 2010:10(2):378-84.

43. Gompert Z, Buerkle CA. A powerful regression-based method for admixture mapping of isolation across the genome of hybrids. Mol Ecol. 2009;18(6):1207-24.

44. Hohenlohe PA, Bassham S, Currey M, Cresko WA. Extensive linkage disequilibrium and parallel adaptive divergence across threespine stickleback genomes. Philos Trans R Soc Lond B Biol Sci. 2012;367(1587): 395-408.

45. Payseur BA, Hoekstra HE. Signatures of reproductive isolation in patterns of single nucleotide diversity across inbred strains of mice. Genetics. 2005; 171(4):1905-16. 
46. Schumer M, Cui R, Powell DL, Dresner R, Rosenthal GG, Andolfatto P. Highresolution mapping reveals hundreds of genetic incompatibilities in hybridizing fish species. Elife. 2014;3:e02535.

47. Jiang CX, Chee PW, Draye X, Morrell PL, Smith CW, Paterson AH. Multilocus interactions restrict gene introgression in interspecific populations of polyploid Gossypium (cotton). Evolution. 2000;54(3):798-814.

48. Reinisch AJ, Dong JM, Brubaker CL, Stelly DM, Wendel JF, Paterson AH. A detailed RFLP map of cotton, Gossypium hirsutum × Gossypium barbadense: chromosome organization and evolution in a disomic polyploid genome. Genetics. 1994;138(3):829-47.

49. Stephens $\mathrm{S}$. The internal mechanism of speciation in Gossypium. Bot Rev. 1950;16(3):115-49.

50. Lopez-Pujol J, Zhang FM, Ge S. No correlation between heterozygosity and vegetative fitness in the narrow endemic and critically endangered Clematis acerifolia (Ranunculaceae). Biochem Genet. 2008:46(7-8):433-45.

Submit your next manuscript to BioMed Central and we will help you at every step:

- We accept pre-submission inquiries

- Our selector tool helps you to find the most relevant journal

- We provide round the clock customer support

- Convenient online submission

- Thorough peer review

- Inclusion in PubMed and all major indexing services

- Maximum visibility for your research

Submit your manuscript at www.biomedcentral.com/submit
Biomed Central 